\title{
Innovación + Educación = Desarrollo (1+E=D)
}

Rafael Antonio Núñez Soriano ${ }^{1}$

En la actualidad se han identificado diversos factores claves para el crecimiento económico de los países en desarrollo, entre los cuales podemos mencionar algunos elementos que no son novedosos para el ser humano desde su creación, porque hablamos de la investigación y la innovación, que son principios que el hombre ha venido implementando a lo largo de la historia; por ejemplo, aprender a trabajar la tierra con sus manos y la domesticación de animales, la fabricaron de automóviles eléctricos usando la tecnología disponible en internet o sensores que trabajan a través de algunas funciones corporales suministrando insulina cuando el cuerpo lo necesita. Pero, la investigación, el desarrollo y la innovación, como conjunto, son conceptos de reciente aparición, aunque son actividades que el ser humano ha venido practicando por muchos años.

Ha sido mediante la innovación y la investigación practicada de manera empírica, que el ser humano desarrolló la mayoría de los descubrimientos que llevaron a la sociedad de niveles de cacería y agricultura simple, hacia un nivel de desarrollo que con el tiempo le facilitó muchos procesos, hasta llegar, a través de los estudios y preparación, a desarrollar la investigación científica que en la actualidad conocemos y de la cual nuestra sociedad goza de muchos beneficios.

El término innovación, según el Diccionario de la Real Academia Española, es la creación o modificación de un producto y su introducción en un mercado. Según Rivas Navarro (2000), ${ }^{2}$ la palabra innovación proviene del latín innovatio, que quiere decir introducir una cosa nueva para reemplazar cualquier otra antigua. Novo (novare) también significa innovar y renovar.

En general, la traducción lleva a concluir que el ser humano observa, analiza y trata de cambiar algo, este puede ser un producto, un servicio, parcial o totalmente para desarrollar una nueva forma o un nuevo artículo que le brinde más comodidad, facilidad o simplemente una sensación nueva y diferente de usarlo.

De acuerdo a diferentes autores, se puede hacer una comparación del por qué la innovación es importante para el desarrollo. Según Peter Drucker (1985): "La

\footnotetext{
1 Universidad Nacional Autónoma de Honduras. Facultad de Ciencias Económicas, Coordinador Académico Departamento de Administración de Empresas. Correo electrónico: rafael.nunez@unah.edu.hn.

${ }^{2}$ Rivas Navarro, M. (2000). Innovación Educativa: Teoría, procesos y estrategias. España: Editorial Síntesis, S.A.

${ }^{3}$ Peter F. Drucker. (1985). The Discipline of Innovation.
} 
innovación crea un recurso que no existe hasta que el hombre encuentra la aplicación de algo natural y entonces la dota de valor económico".

Siguiendo a Drucker, es posible afirmar que la innovación es el medio por el cual una persona o empresa crea nuevos recursos o dota de los recursos existentes con mayor potencial para lograr satisfacer o crear una necesidad en la sociedad; dicho de otra forma, no solamente está relacionada con la creación de nuevos productos y procesos, sino también con la introducción de pequeños cambios que afectan el funcionamiento de los productos ya existentes.

La innovación es sinónimo de desarrollo. Desde la creación del mundo, el hombre innovó en sus cultivos, en sus armas, en su vestido y en cada etapa de la vida estamos innovando de alguna manera; así, los bebes lo hacen en cada momento de su vida, con cada objeto que descubren; cuando somos niños innovamos en los estilos de juego, de aprendizaje; cada uno crea, adapta o desarrolla una forma particular de hacerlo.

El ser humano es innovador por instinto, ya sea en sucesos de buen provecho para la humanidad o en los actos no deseados, como las formas de destruir o hacer daño. Posiblemente, el entorno donde crecemos es determinante para desarrollar en gran medida esta habilidad innata.

Así, durante el desarrollo de la humanidad, los grandes descubrimientos del hombre han sido gracias a esa capacidad o necesidad de innovar; por ejemplo, la creación de los procesos industriales, medios de transporte, de comunicación, etc., cada uno de esos avances ha surgido en la mente de un ser humano por intuición, curiosidad, necesidad o por prueba y error. La innovación no es simplemente un resultado, es parte de un proceso complejo que está compuesto de muchos cambios y actores, que necesita de ciertas y adecuadas condiciones para poder lograr los cambios o mejoras que son acertadas para nuestros deseos 0 necesidades.

\section{Investigación + Desarrollo + innovación (I+D+i)}

En la actualidad cuando se habla de ciencia y tecnología, se escuchamos el concepto I+D+i, que se refiere al proceso mediante el cual los individuos, organizaciones, empresas y países logran desarrollar sus economías por medio de la exploración, diseño, comprobación, implementación, mejora o creación de nuevas formas de hacer las cosas o nuevas maneras de usar ciertos objetos 0 
componentes de los mismos.

Para lograr tener un nivel de medición, cuando hablamos de I+D+i, en un país, se puede conocer la cifra del índice mundial de innovación $(G I I)^{4} 2013$, publicado por el INSEAD The Business School for the World (Escuela de Administración de Empresas al Servicio del Mundo), Universidad Cornell y la Organización Mundial de la Propiedad Intelectual (OMPI).

Es interesante saber que es posible medir a un país por medio de este indicador, pero a la vez es inquietante conocer en qué posición se encuentra nuestro país. Al respecto, de un total de 142 países, Honduras se encuentra en la posición 107, superando solamente a Nicaragua en cuanto a los países de la región y siendo superado por la mayoría de los países de Centroamérica y el Caribe, tal como se observa en la tabla 1.

Tabla 1. Ranking del índice de innovación global de la región Centroamérica y el Caribe $^{5}$

\begin{tabular}{|c|l|c|c|}
\hline Clasificación & \multicolumn{1}{c|}{ País } & Puntuación & $\begin{array}{c}\text { Rango } \\
\text { porcentual }\end{array}$ \\
\hline 39 & Costa Rica & 41.5 & 94.3 \\
\hline 79 & República Dominicana & 33.3 & 80.3 \\
81 & Trinidad y Tobago & 33.2 & 40.4 \\
\hline 82 & Jamaica & 32.9 & 54.6 \\
\hline 86 & Panamá & 31.8 & 10.6 \\
\hline 87 & Guatemala & 31.5 & 53.9 \\
\hline 88 & El Salvador & 31.3 & 43.9 \\
\hline 102 & Belice & 30.0 & 34.7 \\
\hline 107 & Honduras & 28.8 & 19.1 \\
\hline 115 & Nicaragua & 27.1 & 12.0 \\
\hline
\end{tabular}

Según la Organización Mundial de la Propiedad Intelectual (OMPI), el índice mundial de innovación 2013 comprende 142 economías de todo el mundo y en él se utilizan 84 indicadores relativos a la calidad de las principales universidades, la disponibilidad de microfinanciación y los acuerdos de capital de riesgo; indicadores

\footnotetext{
${ }^{4}$ Disponible en: http://globalinnovationindex.org/

${ }^{5}$ The Global Innovation Index 2013. Disponible en: http://globalinnovationindex.org/
} 
mediante los cuales se evalúan las capacidades y los resultados ponderables en el ámbito de la innovación.

El índice mundial de innovación, que se publica anualmente desde 2007, se ha convertido en el principal instrumento de referencia para ejecutivos, políticos y otras personas interesadas en obtener información sobre el estado de la innovación a escala mundial.

Este índice de innovación global ayuda a crear un entorno en donde el factor innovación es continuamente evaluado. Proporciona una herramienta clave y una rica base de datos para 142 economías, que representan el $94,9 \%$ de la población mundial y el $98,7 \%$ del PIB mundial. El estudio de este factor, durante el 2013 , fue focalizado en la función de la ciencia, la tecnología y la innovación y las posibilidades que brinda la cultura para lograr los objetivos de desarrollo del milenio y potenciar el desarrollo sostenible.

De acuerdo a la información que la OMPI publica, en nuestra región tenemos un buen referente, se trata de Costa Rica, que como podemos observar en el gráfico 1 , representa a nivel de toda Latinoamérica una excelente posición.

Gráfico 1. Posicionamiento geográfico del GII 2013 para Latinoamérica

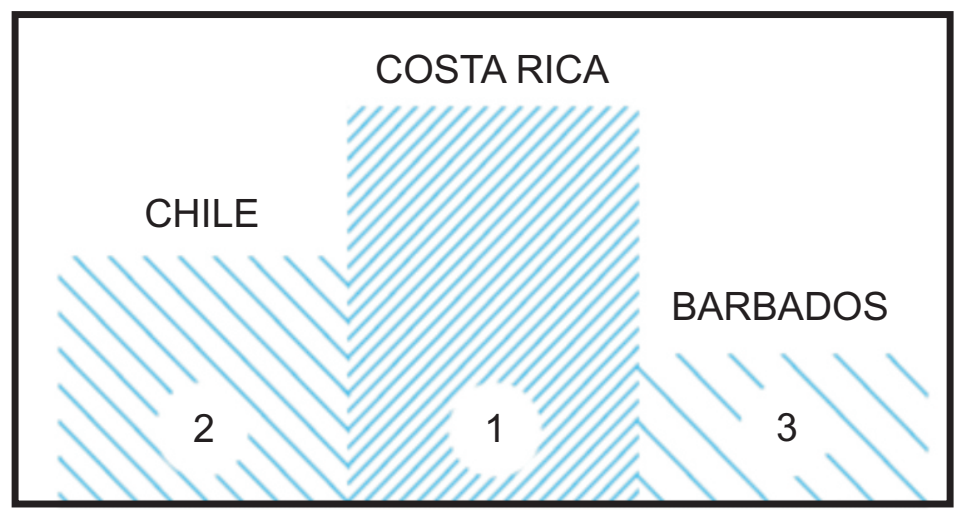

Los datos que presenta el GII, muestran cosas en común entre ciertos países que a pesar de no tener economías tan consolidadas desde hace muchos años, han tenido un despegue considerable en poco tiempo, mejorando notablemente su posición en los índices de desarrollo; en ese grupo podemos mencionar, además de Costa Rica en la región centroamericana, a varios países asiáticos. Todos estos países tienen un común denominador a través del cual han logrado éxito y 
crecimiento económico, se trata del apoyo que sus gobiernos le han brindado a la educación, que es la razón por la que concluimos que la innovación sumada a la educación, nos da como resultado el desarrollo económico y sostenible de las naciones $(I+E=D)$.

La educación científica y el desarrollo de la creatividad, en todos los niveles de escolaridad, desde el sistema básico hasta el superior, es vital para crear 0 desarrollar una sociedad innovadora, por dicha razón vemos la necesidad de mejorar la calidad de los procesos de enseñanza-aprendizaje en todos los niveles educativos, pero, fundamentalmente, en el nivel superior es perentorio hacer una labor de orientación de las habilidades del capital humano, es decir, redirigir los esfuerzos en cada una de las actividades que las carreras actuales están desarrollando, tecnificando, promoviendo y desarrollando la investigación científica y la creatividad para lograr alcanzar índices más altos en cuanto a competitividad e innovación, esto permitirá la creación y sostenibilidad de más centros de investigación y desarrollo tanto en universidades como en instituciones públicas y privadas.

El desafío radica en transformar las instituciones de educación superior (IES) para que estas se enfoquen primordialmente en promover centros de emprendedurismo, incubadoras de empresas, "start-up, spin-off", círculos de creatividad, concursos y ferias de ideas innovadoras de negocios, así como la creación, fortalecimiento y difusión de los Centros de Apoyo a la Tecnología y la Innovación (CATI); todo con el fin de lograr a través del conocimiento que la academia produce y el apoyo de otros sectores, el desarrollo de nuestra sociedad.

La mayoría de los países desarrollan sus economías basados en el conocimiento y parte de estos en las IES. Todas las instituciones educativas del nivel superior se perfeccionan cuando se relacionan con la sociedad, porque tienen nuevas ideas y la industria requiere de nuevas ideas y la sociedad en general les sacara provecho.

Hasta en los últimos años se le ha dado la importancia merecida a la innovación a través de la investigación y otro tipo de proyectos en la educación superior, este tipo de iniciativas ha sentado las bases necesarias para edificar el desarrollo de nuestro país por medio de la ciencia y la tecnología.

Las IES han redefinido su rol en este tema, trazando una nueva y clara visión al concretar la necesidad de una mayor vinculación de los diferentes entes académicos con las empresas y la sociedad, a través de la conformación del Consorcio de Universidades, con el fin de fortalecer la cooperación interuniversitaria 
y fomentar la investigación, desarrollo e innovación. Esta iniciativa de proyecto ha sido promovida por la Dirección de Competitividad e Innovación de la Secretaría de Planificación (SEPLAN).

Además de apoyar la formación del Consorcio de Universidades, el gobierno por medio de SEPLAN y el Consejo Nacional de Competitividad e Innovación (CNCI), recientemente diseñó y publicó la Estrategia Nacional de Competitividad, iniciativa que está orientada a mejorar la situación de Honduras, especialmente a través de dos factores importantes: la eficiencia de los mercados y la innovación productiva.

La Estrategia Nacional de Competitividad tiene como finalidad garantizar la articulación del sector público, el sector privado, la academia, la sociedad civil y los organismos cooperantes, de manera que se involucren integralmente para impulsar la competitividad, la innovación y la mejora de la productividad en nuestro país.

La plataforma para innovar en nuestro país está organizada, pero todavía hay mucho por hacer, ahora necesitamos que los actores entren en acción, se formen, gestionen recursos, unan esfuerzos, conocimientos e inicien una nueva fase y con seguridad encontrarán en las IES lo necesario para poder posicionar a nuestro país en un mejor nivel en el ranking del GII.

Desde la Universidad Nacional Autónoma de Honduras (UNAH), a través de las instancias correspondientes como la Dirección de Investigación Científica, el Instituto de Investigaciones Económicas y Sociales de la Facultad de Economía, el Centro de Emprendedores de la Carrera de Administración de Empresas y otras Direcciones en las diferentes unidades académicas, estamos dispuestos a contribuir con esta transcendental tarea, en beneficio de nuestra querida patria Honduras. 